\title{
Perturbations of Superintegrable Systems
}

\author{
Heinz Hanßmann ${ }^{1}$
}

Received: 25 July 2014 / Accepted: 24 November 2014 / Published online: 23 January 2015

(C) The Author(s) 2015. This article is published with open access at Springerlink.com

\begin{abstract}
A superintegrable system has more integrals of motion than degrees $d$ of freedom. The quasi-periodic motions then spin around tori of dimension $n<d$. Already under integrable perturbations almost all $n$-tori will break up; in the non-degenerate case the resulting $d$-tori have $n$ fast and $d-n$ slow frequencies. Such $d$-parameter families of $d$-tori do survive Hamiltonian perturbations as Cantor families of $d$-tori. A perturbation of a superintegrable system that admits a better approximation by a non-degenerate integrable perturbation of the superintegrable system is said to remove the degeneracy. In the minimal case $d=n+1$ this can be achieved by means of averaging, but the more integrals of motion the superintegrable system admits the more difficult becomes the perturbation analysis.
\end{abstract}

Keywords KAM theory $\cdot$ Ramified torus bundle $\cdot$ Superintegrable system $\cdot$ Euler top · Central force field $\cdot$ Non-integrable resonances $\cdot$ C. Neumann hierarchy

\section{Introduction}

Integrable Hamiltonian systems are in $d \geq 2$ degrees of freedom the exception rather than the rule. This makes the behaviour of the dynamics under non-integrable perturbations one of the key properties of an integrable system. On a general level one obtains information on motions of an open subset of all Hamiltonian systems [32], and for concretely given systems one can address the question how the dynamics organizes the phases space.

The typical bounded motion in an integrable system is quasi-periodic, spinning densely around invariant tori. This makes the phase space a ramified torus bundle, the regular fibres of $n$-tori forming families that are organized by families of invariant $(n-1)$-tori, which in turn are organized by bifurcating $(n-1)$-tori and by $(n-2)$-tori and so on, down to the equilibria of the Hamiltonian system. In the case $n=d$ of Lagrangean tori one can apply KAM theory directly to the integrable system, yielding persistence of the Lagrangean tori $[2,8,20]$, of elliptic/hyperbolic lower dimensional tori $[7,8,35]$ and of invariant tori

1 Mathematisch Instituut, Universiteit Utrecht, 3508 TA Utrecht, The Netherlands 
that undergo bifurcations $[10,28,29]$. Such results are valid under non-degeneracy conditions [30], the best-known being Kolmogorov's non-degeneracy condition which expresses that the frequency mapping from the families of Lagrangean tori to $\mathbb{R}^{d}$ is a submersion.

Superintegrable systems fail to satisfy such non-degeneracy conditions on a fundamental level and are consequently termed properly degenerate systems in [3]. Famous examples are the 2-body problem, resonant oscillators, rigid bodies fixed at their centre of mass and combinations of these; in particular the Kepler approximation of the $n$-body problem, with all planets only interacting with the sun and not with each other.

The additional integrals of motion of a superintegrable system simplify the analysis of the (unperturbed) dynamics. For instance, in the maximally superintegrable case of $2 d-1$ conserved quantities the regular fibres of the ramified torus bundle are merely periodic orbits (or circles of equilibria) organized by bifurcating periodic orbits and by (isolated) equilibria. However, the price to pay is that the perturbation analysis becomes more complicated and involves the perturbation as well. In the extreme case of a maximally superintegrable system one has to study the Hamiltonian system in $d-1$ degrees of freedom that is obtained by averaging the perturbation along the unperturbed periodic orbits and reducing the acquired $S^{1}$-symmetry.

A Hamiltonian system that is closer to the perturbed system than the unperturbed superintegrable system is called an intermediate system if it has additional integrals of motion compared to the perturbed system but less integrals of motion than the unperturbed system. Normal forms provide the means to obtain intermediate systems, and the most obvious way is to normalize by averaging along the unperturbed dynamics. In general such an intermediate system inherits $n+1$ integrals of motion in involution and the perturbation is said to remove the degeneracy [3] in case of a (non-degenerate) integrable intermediate system.

In the minimally superintegrable case $d=n+1$ the intermediate system is automatically integrable. Between these two extreme cases there is a whole hierarchy of superintegrable systems with $2 d-n$ independent conserved quantities, $n=1, \ldots, d-1$. Reducing the acquired $\mathbb{T}^{n}$-symmetry leads to $d-n$ degrees of freedom and in general one is back to studying a general Hamiltonian system (albeit with fewer degrees of freedom). A general statement of quasi-periodic persistence is proven in [3] under the condition that the reduced intermediate system is a non-degenerate integrable system and the frequency mapping of the superintegrable system onto $\mathbb{R}^{n}$ is a submersion as well.

This review is organized as follows. Section 2 contains the behaviour of the unperturbed system, in particular the geometry imposed by the dynamics. In Sect. 3 the treatment of perturbed minimally superintegrable systems is exemplified for the Euler top and the logarithmic potential. Section 4 is devoted to resonant oscillators, where superintegrability may or may not be minimal, and more examples of the latter are given in Sect. 5. The final conclusions constitute Sect. 6.

\section{Unperturbed Dynamics}

Let $(\mathscr{P}, \sigma)$ be a symplectic manifold of dimension $\operatorname{dim} \mathscr{P}=2 d$ and define first Hamiltonian vector fields by means of $\sigma\left(X_{H}, Y\right)=\mathrm{d} H(Y)$ for all vector fields $Y$ on $\mathscr{P}$ and then the Poisson bracket $\{f, g\}:=-\sigma\left(X_{f}, X_{g}\right)$.

The simplest way to define a superintegrable system is to require it to be an integrable Hamiltonian system, with $d$ integrals of motion in involution $\left\{I_{k}, I_{\ell}\right\}=0$ and admitting at least one more conserved quantity independent of $I_{1}, \ldots, I_{d}$. Since the Poisson bracket of two conserved quantities is again an integral of motion all these form a Lie algebra, 
and as the symplectic structure is non-degenerate such a Lie algebra is necessarily noncommutative once the dimension exceeds $d$. One therefore also speaks of non-commutative integrabililty [34]. The following result from [23] clarifies the semi-local situation (i.e. locally around a maximal torus).

Theorem 1 On the subset $\mathscr{M} \subseteq \mathscr{P}$ of the phase space with symplectic structure $\sigma$ let $f$ : $\mathscr{M} \longrightarrow \mathscr{B} \subseteq \mathbb{R}^{2 d-n}$ be a submersion with compact and connected fibres (hence, a fibration). Assume that $\left\{f_{i}, f_{j}\right\}=P_{i j} \circ f, i, j=1, \ldots, 2 d-n$ and that the matrix $P$ with entries $P_{i j}: \mathscr{M} \longrightarrow \mathbb{R}$ has rank $2(d-n)$ at all points of $\mathscr{B}$. Then every fibre of $f$ is diffeomorphic to $\mathbb{T}^{n}$ and the fibration $f$ has local trivialisations which are symplectic.

Taking for $\mathscr{M}$ the regular part of the phase space where the integrals are independent and have compact (common) level sets, every fibre of $f$ has a neighbourhood $\mathscr{U}$ with coordinates

$$
(x, y, q, p): \mathscr{U} \quad \longrightarrow \quad \mathbb{T}^{n} \times \mathbb{R}^{n} \times \mathbb{R}^{d-n} \times \mathbb{R}^{d-n}
$$

such that the level sets of $f$ coincide with the level sets of $(y, q, p)$ and

$$
\sigma_{\mid \mathscr{U}}=\sum_{k=1}^{n} \mathrm{~d} x_{k} \wedge \mathrm{d} y_{k}+\sum_{\ell=1}^{d-n} \mathrm{~d} q_{\ell} \wedge \mathrm{d} p_{\ell} .
$$

These co-ordinates are Nekhoroshev's generalized action angle variables [36]. In particular we see that locally we have $d$ commuting integrals of motion $y_{1}, \ldots, y_{n}, p_{1}, \ldots, p_{d-n}$ and $d-n$ additional conserved quantities $q_{1}, \ldots, q_{d-n}$ that are in involution with the first $n$ integrals $y_{1}, \ldots, y_{n}$.

On $\mathscr{M}$ the superintegrable system is equivariant with respect to the $\mathbb{T}^{n}$-action $x \mapsto x+$ $\xi$ and in examples this action often extends to all of $\mathscr{P}$ (simple counter-examples with $n=1$ can be constructed from Hamiltonian systems in one degree of freedom). Reduction of such a $\mathbb{T}^{n}$-action leads to $d-n$ degrees of freedom where $(q, p)$ provide canonical coordinates. This can in particular be applied to an intermediate system obtained from a given perturbation by averaging over the unperturbed fast motion.

The perturbation is said to remove the degeneracy (of the superintegrable or 'properly degenerate' unperturbed system) if the intermediate system is indeed integrable. Then we may choose $(q, p)$ to be action angle variables of the reduced intermediate system and the Lagrangean tori

$$
\mathbb{T}^{n} \times \mathbb{T}^{d-n} \times\{y\} \times\{p\}
$$

with angular variables $(x, q)$ have frequency vectors

$$
\omega(y, p)=\left(\omega_{1}(y), \ldots, \omega_{n}(y), \omega_{n+1}(y, p), \ldots, \omega_{d}(y, p)\right)
$$

where the last $d-n$ components - those that do depend on the variables $p_{1}, \ldots, p_{d-n}$-are of the order of the perturbation (while $\omega_{1}, \ldots, \omega_{n}$ are of order one). Non-degeneracy of the frequency mapping

$$
\omega: \mathscr{M} \quad \longrightarrow \quad \mathbb{R}^{d}=\mathbb{R}^{n} \times \mathbb{R}^{d-n}
$$

defined by (2) then yields Cantor families of invariant Lagrangean tori in the (original) perturbed system, cf. [3, 4]. 
The leaves of the fibration $f: \mathscr{M} \longrightarrow \mathscr{B}$ are $n$-tori on which the symplectic structure vanishes; one also speaks of an isotropic fibration [23]. The symplectic leaves of the base space $\mathscr{B}$ have dimension $d-n$ and constitute the phase space for the reduced intermediate system. In case these define a fibration $s: \mathscr{B} \longrightarrow \mathscr{A}$ the composition $s \circ f: \mathscr{M} \longrightarrow \mathscr{A}$ is the co-isotropic fibration $[16,23]$ and the actions $y_{1}, \ldots, y_{n}$ conjugate to the toral angles are local co-ordinates on the base space $\mathscr{A}$.

The fibres of the co-isotropic fibration are the Fassò flowers [22, 23] and form themselves fibrations $f_{y}: f^{-1}\left(s^{-1}(y)\right) \longrightarrow s^{-1}(y), y \in \mathscr{A}$. The symplectic leaf $s^{-1}(y)$ is the centre of the flower and the isotropic $n$-tori $f^{-1}(z), z \in s^{-1}(y)$ are the petals, while the action manifold $\mathscr{A}$ is the meadow on which the flowers grow. The bifibration $\mathscr{M} \rightarrow \mathscr{B} \rightarrow \mathscr{A}$ allows to distinguish between semi-local properties (in a neighbourhood of an $n$-torus) and semi-global properties (in a neighbourhood of a flower). In particular, the generalized action angle variables (1) are semi-local co-ordinates, while a semi-global chart of $\mathscr{M}$ would also be global onto (a model space for) the symplectic fibres of $\mathscr{B}$.

\section{Minimally Superintegrable Systems}

The intermediate system is always integrable if $d-n=1$ and $\partial_{p} \omega_{d}(y, p) \neq 0$ is all that is needed for (2) to be non-degenerate (provided that the superintegrable frequency mapping $y \mapsto\left(\omega_{1}(y), \ldots, \omega_{n}(y)\right)$ is non-degenerate). This is a genericity condition on the perturbation which can be explicitly checked after averaging over the unperturbed fast motion. For a concretely given system the task is therefore to fully analyse the one-degree-of-freedom problem parametrised by $n$ actions - and thus identifying how the families of $d$-tori are organized by the fast $n$-tori and their bifurcations.

Both examples in this section have $d=3$ degrees of freedom and are superintegrable because the energy $H$ and the three components $\mu_{1}, \mu_{2}, \mu_{3}$ of the angular momentum are conserved quantities. Replacing one of the components, say $\mu_{1}$, by the total angular momentum $|\mu|=\sqrt{\mu_{1}^{2}+\mu_{2}^{2}+\mu_{3}^{2}}$ yields three commuting integrals $H,|\mu|$ and, say, $\mu_{3}$ with the fourth integral $\mu_{2}$ commuting with $H$ and $|\mu|$ (but not with $\mu_{3}$ ).

Note that there are moreover invariant lower dimensional tori in the intermediate system that have one slow frequency, these come from the singular fibres of the ramified torus bundle defined by the unperturbed dynamics. The symplectic normal behaviour of such a torus, and whether this undergoes a bifurcation, is then decided by the fast dynamics as well. Also the combination of e.g. two bifurcations in both the fast and the slow dynamics is possible, with resulting phenomena in $1+1$ rather than truly 2 degrees of freedom (for co-dimension 1 bifurcations).

\subsection{The Euler Top}

The configuration space of a rigid body with a fixed point is $S O(3)$, whence we have $\mathscr{P}=$ $T^{*} S O(3)$ for the phase space. In the absence of external forces or torques the system is invariant under the left action of $S O(3)$ - the lift of $(g, h) \mapsto g h$. The components $\ell_{1}, \ell_{2}, \ell_{3}$ of the angular momentum with respect to the body axes transform as $\ell \mapsto g^{-1}(\ell)$ under the right action— the lift of $(g, h) \mapsto h g$ - whence the (kinetic) energy

$$
H_{0}(g, \ell)=\frac{\ell_{1}^{2}}{2 I_{1}}+\frac{\ell_{2}^{2}}{2 I_{2}}+\frac{\ell_{3}^{2}}{2 I_{3}}
$$


is invariant under the left action but not invariant under the right action. Here $I_{1}, I_{2}, I_{3}$ are the principal moments of inertia, the body set of axes been chosen along the principal axes of inertia. Still, the $S O(3)$-symmetry from the left action makes the components $\mu_{1}, \mu_{2}, \mu_{3}$ of the angular momentum with respect to the spatial axes conserved quantities, next to the Hamiltonian $H_{0}$.

The spatial components of the angular momentum constitute the momentum mapping $J$ of the left $S O(3)$ action and the isotropy group $G_{\mu}$ of the co-adjoint action of $S O(3)$ on $\mathfrak{s o}(3)^{*} \cong \mathbb{R}^{3}$ consists of the rotations about $0 \neq \mu \in \mathbb{R}^{3}$. Reducing the $S O(3)$-symmetry leads to a one-degree-of-freedom system on the co-adjoint orbit

$$
J^{-1}(\mu) /_{G_{\mu}} \cong S O(3) /_{S^{1}} \cong S_{|\mu|}^{2}=\left\{\left.\ell \in \mathbb{R}^{3}\left|\ell_{1}^{2}+\ell_{2}^{2}+\ell_{3}^{2}=\right| \mu\right|^{2}\right\} .
$$

Action angle variables $\left(x_{1}, y_{1}\right)$ of this integrable system serve as starting point for the construction of generalized action angle variables for the free rigid body. The second angle $x_{2}$ measures the rotation (= precession) about the angular momentum vector, with conjugate action $y_{2}=|\mu|$. The phase space $\mathscr{P}=T^{*} S O(3)$ has left trivialization

$$
\begin{aligned}
\left(\pi, T_{e}^{*} L_{g}\right): T^{*} S O(3) & \longrightarrow S O(3) \times \mathbb{R}^{3} \\
\alpha_{g} & \mapsto(g, \ell)
\end{aligned}
$$

and right trivialization

$$
\begin{aligned}
\left(T_{e}^{*} R_{g}, \pi\right): T^{*} S O(3) & \longrightarrow \mathbb{R}^{3} \times S O(3) \\
\alpha_{g} & \mapsto(\mu, g)
\end{aligned}
$$

where $\pi: T^{*} S O(3) \longrightarrow S O(3)$ is the bundle projection. In the right trivialization $x_{1}, x_{2}$ and $y_{1}$ are co-ordinates on $S O(3)$ and $y_{2}$ fixes the radius of the sphere

$$
\left\{\mu \in \mathbb{R}^{3} \mid \mu_{1}^{2}+\mu_{2}^{2}+\mu_{3}^{2}=y_{2}^{2}\right\} .
$$

Choosing canonical co-ordinates $(q, p)$ on the latter yields generalized action angle variables $(x, y, q, p)$, while $(x, y, \mu)$ define semi-global charts when restricting $\mu$ to (4).

The angle $x_{2}$ is not globally defined since $S O(3) \rightarrow S^{2}$ is not a trivial $S^{1}$-bundle, being related to the Hopf fibration. In the dynamically symmetric case $I_{1}=I_{2} \neq I_{3}$ we may use $y_{1}=\ell_{3}$ and the action angle variables $\left(x_{1}, y_{1}\right)$ are cylindrical co-ordinates on the 'right' sphere (3), this is the situation sketched in Fig. 1. Choosing also cylindrical co-ordinates $(q, p)$ on the 'left' sphere (4) yields the Andoyer variables $(x, y, q, p)$. Note that

$$
H_{0}(y)=\frac{y_{2}^{2}}{2 I_{1}}-\frac{I_{3}-I_{1}}{I_{1} I_{3}} \frac{y_{1}^{2}}{2}
$$

in the dynamically symmetric case whence

$$
\operatorname{det} D^{2} H_{0}(y)=-\frac{I_{3}-I_{1}}{I_{1}^{2} I_{3}} \neq 0
$$

shows that the frequency mapping is a submersion.

The $\mathbb{T}^{2}$-action $x \mapsto x+\xi$ is globally defined in the dynamically symmetric case, except for the zero-section $S O(3) \subseteq T^{*} S O(3)$ of the phase space. Furthermore, the elliptic functions 


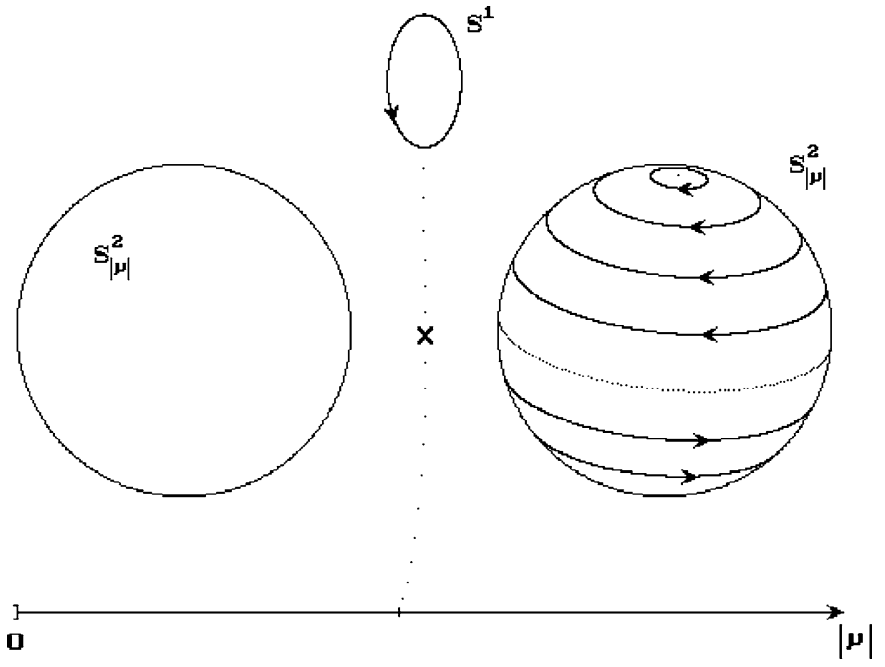

Fig. 1 Schematic representation of the bundle structure of the phase space $\mathscr{P}=T^{*} S O(3)$. The value $y_{2}=|\mu|=0$ is excluded from the picture, in this limit the product of the spheres with $S^{1}$ attached results in a fibre $S O(3)$

solving $\dot{\ell}=\ell \times I^{-1}(\ell)$ become sines and cosines (the rotation along the angle $x_{1}$ measures the spinning of the rigid body) and the intermediate system can be computed by hand. For instance, averaging an affine force field [27] along the Eulerian tori yields

$$
\begin{aligned}
\bar{H}(y, \mu)= & H_{0}(y)+\frac{a+b+c}{2}\left(I_{1}+\left(I_{3}-I_{1}\right) \frac{y_{1}^{2}}{y_{2}^{2}}\right) \\
& +s_{3} \frac{y_{1}}{y_{2}^{2}}\left(\alpha \mu_{1}+\beta \mu_{2}+\gamma \mu_{3}\right)-\frac{I_{3}-I_{1}}{2} \frac{3 y_{1}^{2}-y_{2}^{2}}{y_{2}^{4}}\left(a \mu_{1}^{2}+b \mu_{2}^{2}+c \mu_{3}^{2}\right)
\end{aligned}
$$

where $s_{3}$ is the component of the centre of mass along the third body axis and the other coefficients come from the (small) potential $V=\alpha x+\beta y+\gamma z+a x^{2}+b y^{2}+c z^{2}$ of the affine force field.

The phase space structure of the intermediate system can be read off from the slice $y_{2}=|\mu|$ of the set of singular values of the energy-momentum mapping, see Fig. 2. For values of $y_{1}$ near $\pm \frac{1}{3}|\mu|$ the linear part of the force field has negligeable influence and the angular momentum rotates (= nutates) about the direction of the constant part of the force field. Lowering the value of $\left|y_{1}\right|$ leads to two successive centre-saddle bifurcations and when $y_{1}$ passes through 0 the two resulting saddles undergo a connection bifurcation (where only the linear part of the force field contributes, the saddles are connected by heteroclinic orbits).

There is no centre-saddle bifurcation for $\left|y_{1}\right|$ increasing from $\frac{1}{3}|\mu|$ if the constant part of the force field dominates the linear part while both centre-saddle bifurcations also take place for $\left|y_{1}\right|$ increasing from $\frac{1}{3}|\mu|$ to $|\mu|$ if the constant part of the force field is itself dominated by the linear part. Figure 2 depicts the middle case where both parts of the affine force field are of comparable influence, then only the first centre-saddle bifurcation occurs as $\left|y_{1}\right|$ comes close to $|\mu|$. For fixed values of $y$ the Eulerian tori and their separatrices thus organize the distribution of 3-tori into 1-5 families. Most of the invariant 3-tori persist under the perturbation from the intermediate system back to the original rigid body subject to a 


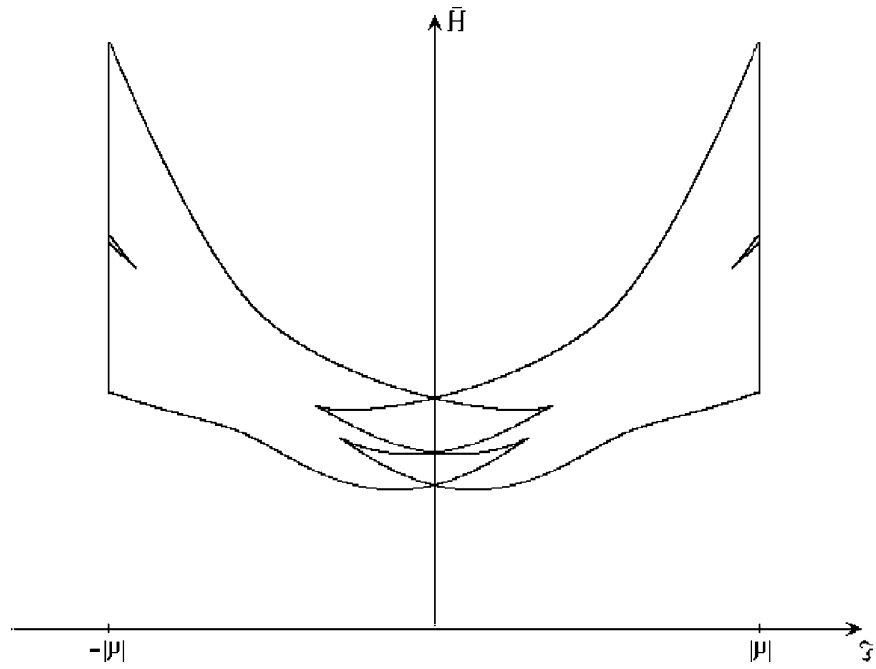

Fig. 2 A slice $y_{2}=|\mu|$ of the set of critical values of the energy-momentum mapping $\mathscr{E} \mathscr{M}=(y, \bar{H})$ with axes $y_{1}=\ell_{3}$ and $\bar{H}$ for the 'middle' case $s_{3} \cdot(\alpha, \beta, \gamma) \approx(a, b, c)$

weak affine force field [33]. That the necessary non-degeneracy condition holds trueslow frequency varies from torus to torus-is in fact enforced by the way the Eulerian tori organize the phase space.

In the intermediate system the connection bifurcation takes place exactly at the strong resonance $\omega_{1}(y)=0$ of the Eulerian tori, see Fig. 1. Also the centre-saddle bifurcations do not persist by general theory [29]. Indeed, the same ratio $\frac{y_{1}}{y_{2}}$ that parametrises the lines of parabolic Eulerian tori also fixes the frequency ratio $\frac{\omega_{1}}{\omega_{2}}=\frac{I_{3}-I_{1}}{I_{3}} \frac{y_{1}}{y_{2}}$ and the whole bifurcation scenario may fall into a resonance gap. However, for most values of parameters $s_{3}, I_{1}, I_{3}$ the quasi-periodic centre-saddle bifurcations turn out to be present in the original dynamics [28].

\subsection{The Logarithmic Potential}

The motion of a mass point subject to a central force field provides for a whole class of Hamiltonian systems with $S O(3)$-symmetry; here the natural action on the configuration space $\mathbb{R}^{3}$ is lifted to the diagonal action on the phase space $T^{*} \mathbb{R}^{3}=\mathbb{R}^{3} \times \mathbb{R}^{3}$. Again the components $\mu_{1}, \mu_{2}, \mu_{3}$ of the angular momentum are conserved quantities, next to the Hamiltonian $H_{0}$. To fix thoughts we concentrate on the case

$$
H(\xi, \eta)=\frac{\eta_{1}^{2}+\eta_{2}^{2}+\eta_{3}^{2}}{2}+\frac{1}{2} \ln \left(R^{2}+\xi_{1}^{2}+\xi_{2}^{2}+\xi_{3}^{2}\right)
$$

of logarithmic potentials, where we scaled the mass to 1 . Note that the origin $(\xi, \eta)=(0,0)$ of the phase space is an equlibrium for $R>0$, while in the so-called scale-free case $R=0$ the potential is not defined at the origin $\xi=0$ of the configuration space.

The components of the angular momentum constitute the momentum mapping $J$ of the $S O(3)$ action and the isotropy group $G_{\mu}$ of the co-adjoint action of $S O(3)$ on $\mathfrak{s o}(3)^{*} \cong \mathbb{R}^{3}$ consists of the rotations about $0 \neq \mu \in \mathbb{R}^{3}$. Reducing the $S O(3)$-symmetry leads to a one- 
degree-of-freedom system on

$$
J^{-1}(\mu) /_{G_{\mu}} \cong\left\{(q, p) \in \mathbb{R}^{4}\left|q_{1} p_{2}-q_{2} p_{1}=\right| \mu \mid\right\} / S^{1}
$$

where $(q, p) \in T^{*} \mathbb{R}^{2}$ are linear variables on the plane perpendicular to $\mu$ in which the motion takes place. The diagonal $S^{1}$-action on the latter can be factored out using the invariants

$$
u=\frac{q_{1}^{2}+q_{2}^{2}}{2}, \quad v=\frac{p_{1}^{2}+p_{2}^{2}}{2}, \quad w=q_{1} p_{1}+q_{2} p_{2}
$$

which together with $|\mu|=q_{1} p_{2}-q_{2} p_{1}$ generate the ring of $S^{1}$-invariant functions on $T^{*} \mathbb{R}^{2}=\mathbb{R}^{2} \times \mathbb{R}^{2}$. This turns (5) into

$$
\mathscr{P}_{|\mu|}=\left\{(u, v, w) \in \mathbb{R}^{3} \mid u \geq 0, v \geq 0, S_{|\mu|}(u, v, w)=0\right\}
$$

where the syzygy

$$
S_{|\mu|}(u, v, w)=\frac{w^{2}}{2}-2 u v+\frac{|\mu|^{2}}{2}
$$

between the invariants also allows to write down the Poisson bracket

$$
\{f, g\}=\left\langle\nabla f \times \nabla g \mid \nabla S_{|\mu|}\right\rangle
$$

on $\mathbb{R}^{3}$ and thus on $\mathscr{P}_{|\mu|}$. Alternatively, one can work with the effective potential

$$
V_{|\mu|}(u)=\frac{1}{2} \ln \left(R^{2}+2 u\right)+\frac{|\mu|^{2}}{4 u} .
$$

Action angle variables $\left(x_{1}, y_{1}\right)$ of the resulting one-degree-of-freedom system serve as starting point for the construction of generalized action angle variables. The second angle $x_{2}$ measures the rotation about the angular momentum vector, with conjugate action $y_{2}=|\mu|$. Restricting $\mu \in \mathbb{R}^{3}$ to the sphere

$$
\left\{\mu \in \mathbb{R}^{3} \mid \mu_{1}^{2}+\mu_{2}^{2}+\mu_{3}^{2}=y_{2}^{2}\right\}
$$

yields a semi-global chart $(x, y, \mu)$, while any choice of canonical co-ordinates on (6) defines generalized action angle variables for $H_{0}$. The Hamiltonian

$$
H(\xi, \eta)=\frac{\eta_{1}^{2}+\eta_{2}^{2}+\eta_{3}^{2}}{2}-\Omega\left(\xi_{1} \eta_{2}-\xi_{2} \eta_{1}\right)+\frac{1}{2} \ln \left(R^{2}+\frac{\xi_{1}^{2}}{\rho_{1}^{2}}+\frac{\xi_{2}^{2}}{\rho_{2}^{2}}+\frac{\xi_{3}^{2}}{\rho_{3}^{2}}\right)
$$

models a simple but realistic 3-dimensional matter distribution, with reflectional symmetries with respect to the principal planes of the system and (for $\Omega \neq 0$ ) overall bulk rotation. The scale-factors $\rho_{k}$ describing the axial ratios of the equipotential surfaces are close to each other, i.e. the differences between the $\rho_{k}$ are small, and the angular velocity $\Omega$ of the rotating galaxy is a small parameter as well. The astrophysical relevance of the model $[5,6]$ is based on its ability to describe in a simple way the gross features of elliptic galaxies embedded in a dark matter halo. It has therefore been the subject of several applications in galactic dynamics [24, 38, 43].

Reducing the $\mathbb{T}^{2}$-symmetry of the intermediate system leads to a one-degree-of-freedom problem on (6). Its dynamics describes the slow (periodic) variation of the $(q, p)$-plane 
within which the fast rosetta-orbits of $H_{0}$ take place. These invariant 3-tori are organized by the equilibria of the one-degree-of-freedom problem-the surviving fast 2-tori-and their bifurcations.

Persistence of invariant 3-tori when perturbing from the intermediate system back to the original (7) relies on separate non-degeneracy conditions on the slow and fast frequencies. The numerical results in [41] indicate that the latter are iso-energetically non-degenerate, with a frequency ratio that decreases monotonically under variation of $y_{2}$. The 3-tori in [37] are computed for such high values of $\rho_{k}-\rho_{\ell}$ (while $\Omega=0$ ) that the distinction between slow and fast frequencies disappears and low order resonances emerge.

\section{Resonant Equilibria}

An elliptic equilibrium of a Hamiltonian system has all eigenvalues on the imaginary axis but no multiple eigenvalues. In particular 0 is not an eigenvalue, the equilibrium persists and all eigenvalues remain on the imaginary axis if the system is slightly perturbed. The quadratic part

$$
H_{\alpha}^{2}(q, p)=\sum_{\ell=1}^{d} \alpha_{\ell} \frac{p_{\ell}^{2}+q_{\ell}^{2}}{2}
$$

may have imaginary part $\alpha_{\ell}$ of the eigenvalues of both signs-since the equilibrium does not need to be a local extremum of the Hamiltonian it is not possible to enforce $\alpha_{\ell}>0$ for all $\ell$ - the so-called symplectic sign.

One speaks of a resonance between the normal frequencies $\alpha_{1}, \ldots, \alpha_{d}$ if there are integers $k_{1}, \ldots, k_{d} \in \mathbb{Z}$, not all zero, such that

$$
k_{1} \alpha_{1}+\cdots+k_{d} \alpha_{d}=0
$$

Here $k_{1}, \ldots, k_{d}$ can be taken relatively prime and $|k|=\left|k_{1}\right|+\cdots+\left|k_{d}\right|$ is called the order of the resonance. Since we excluded the $1: 0$ and $1: \pm 1$ resonances we have $|k| \geq 3$. If there are no resonances of order $|k| \leq m$, then the normal form truncated at order $m$ depends on $(q, p)$ only as a function of the invariants

$$
I_{\ell}=\frac{p_{\ell}^{2}+q_{\ell}^{2}}{2} \geq 0
$$

whence we obtain for $m=4$ the (truncated) Birkhoff normal form

$$
\mathscr{H}_{\alpha, \beta}(I)=H_{\alpha}^{2}(I)+\sum_{k, \ell=1}^{d} \beta_{k \ell} \frac{I_{k} I_{\ell}}{2}
$$

with a symmetric matrix $\beta=\left(\beta_{k \ell}\right)_{k, \ell=1, \ldots, d}$. This normal form $\mathscr{H}_{\alpha, \beta}$ is (near the equilibrium) an integrable approximation of the original Hamiltonian and under $e$.g. Kolmogorov's nondegeneracy condition $\operatorname{det} \beta \neq 0$ a measure-theoretically large part of a neighbourhood of the equilibrium is foliated by a Cantor family of invariant $d$-tori [4].

The structure of the ramified torus bundle defined by the dynamics of $\mathscr{H}_{\alpha, \beta}$ is very transparant. The values $I_{\ell}>0$ parametrise the periodic orbits in the $\left(q_{\ell}, p_{\ell}\right)$-plane, where $I_{1}, \ldots, I_{\ell-1}, I_{\ell+1}, \ldots, I_{d}$ vanish. Taking $n \geq 2$ among the $I_{\ell}$ to be non-zero parametrises the 
various $n$-parameter families of invariant $n$-tori, up until the single $d$-parameter family of Lagrangean tori.

Liapunov's Centre Theorem yields persistence of those $\left(q_{\ell}, p_{\ell}\right)$-planes of periodic orbits for which none of the other frequencies is an integer multiple of $\alpha_{\ell}$. Persistence of $n$-tori with $2 \leq n \leq d-1$, parametrised by, say, $I_{1}, \ldots, I_{n}$, requires non-degeneracy conditions on the frequencies $\omega_{\ell}(I)=\alpha_{\ell}+\beta_{\ell 1} I_{1}+\cdots+\beta_{\ell n} I_{n}$ that ensure the Diophantine conditions

$$
\left|k_{1} \omega_{1}(I)+\cdots+k_{n} \omega_{n}(I)+k_{n+1} \alpha_{n+1}+\cdots+k_{d} \alpha_{d}\right| \geq \frac{\gamma}{|k|^{\tau}}
$$

to hold true for all $k_{1}, \ldots, k_{n} \in \mathbb{Z}$ (not all zero) and $k_{n+1}, \ldots, k_{d} \in\{0, \pm 1, \pm 2\}$ on large Cantor sets in $I$-space $\mathbb{R}^{n}$.

\subsection{Integrable Resonances}

The resonances (9) of order $|k| \leq 4$ read

$$
\alpha_{\ell}= \pm 2 \alpha_{j}, \quad \alpha_{\ell}+\alpha_{j}= \pm \alpha_{i}
$$

and

$$
\alpha_{\ell}= \pm 3 \alpha_{j}, \quad \alpha_{\ell}= \pm \alpha_{j} \pm 2 \alpha_{i}, \quad \alpha_{\ell}+\alpha_{j}= \pm \alpha_{i} \pm \alpha_{m}
$$

and if we also allow for multiple (but non-zero) eigenvalues on the imaginary axis we furthermore have

$$
\alpha_{\ell}= \pm \alpha_{j}
$$

In case of a single low order resonance the Hamiltonian (8) is superintegrable with quasiperiodic motions spinning densely around $(d-1)$-tori. To fix thoughts let the resonance read

$$
\alpha_{1}= \pm k \alpha_{2}, \quad k \in\{2,3\}
$$

whence the real and imaginary parts of

$$
J=\frac{1}{k !}\left(q_{1} \pm \mathrm{i} p_{1}\right)\left(q_{2}-\mathrm{i} p_{2}\right)^{k}
$$

are among the generators of the $\mathbb{T}^{d-1}$-symmetric functions, leading to the syzygy

$$
\frac{|J|^{2}}{2}=\frac{2^{k}}{k !} I_{1} I_{2}^{k} .
$$

Note that

$$
K:= \pm k I_{1}+I_{2}
$$

has vanishing Poisson bracket not only with $I_{1}, \ldots, I_{d}$, but also with

$$
u:=\operatorname{Re} J, \quad v:=\operatorname{Im} J
$$

and with

$$
w:=\mp k I_{1}+I_{2}
$$


Fixing $y_{1}=K$ the syzygy (12) reads

$$
S_{y}(u, v, w)=\frac{u^{2}+v^{2}}{2} \pm \frac{\left(w-y_{1}\right)\left(w+y_{1}\right)^{k}}{2 k \cdot k !}=0
$$

and together with the actions $y_{\ell}:=I_{\ell+1}, \ell=2, \ldots, d-1$ and conjugate angles $x_{\ell}, \ell=$ $1, \ldots, d-1$ we obtain a semi-global chart $(x, y, u, v, w)$ when restricting $(u, v, w)$ to

$$
\mathscr{P}_{y}:=\left\{(u, v, w) \in \mathbb{R}^{3} \mid-y_{1} \leq w \leq y_{1}, S_{y}(u, v, w)=0\right\}
$$

in the definite case $\alpha_{1}=k \alpha_{2}$ and to

$$
\mathscr{P}_{y}:=\left\{(u, v, w) \in \mathbb{R}^{3}|w \geq| y_{1} \mid, S_{y}(u, v, w)=0\right\}
$$

in the indefinite case $\alpha_{1}=-k \alpha_{2}$ (recall $k \in\{2,3\}$ ). Any choice of canonical co-ordinates on $\mathscr{P}_{y}$ yields generalized action angle variables.

For a single resonance the intermediate system is given by the truncated Gustavson or resonant (Birkhoff) normal form

$$
\mathscr{H}_{\alpha, \lambda}(y, u, v, w)=H_{\alpha}^{2}(y)+\lambda w+\gamma(\lambda) u+\sum_{j, \ell=1}^{d} \beta_{j \ell}(\lambda) \frac{y_{j} y_{\ell}}{2}
$$

of order 4 , where $\lambda$ is the so-called detuning parameter and we abused notation by letting $y_{d}=w$ in the final sum. Note that the (linear) term in $v$ has been removed by means of a $\lambda$-dependent rotation in the $(u, v)$-plane [17, 31].

Reducing the $\mathbb{T}^{d-1}$-symmetry of the intermediate system leads to a one-degree-offreedom problem on $\mathscr{P}_{y}$ that determines how the invariant $d$-tori are organized into $d$-parameter families. The resulting ramified torus bundle has $d-1$ fast frequencies (in the angles $x_{1}, \ldots, x_{d-1}$ ) and one slow frequency (for $y_{1}$ and $\lambda$ sufficiently small). The final step then is to apply KAM theory to obtain Cantor sub-families of invariant tori that survive the perturbation back to the original Hamiltonian.

\subsection{Non-integrable Resonances}

For two or more resonances (9) the resonant Birkhoff or Gustavson normal form is no longer guaranteed to be integrable. Writing $d-n$ for the number of independent resonances, the superintegrable system of coupled harmonic oscillators (8) defines a ramified $\mathbb{T}^{n}$-bundle, the geometry of which depends on the resonances at hand. In particular the flow is periodic in the maximal case of $d-1$ resonances. Let us restrict to $d=3$ coupled oscillators whence scaling time allows to rewrite (8) as

$$
H_{m}^{2}(q, p)=m_{1} I_{1}+m_{2} I_{2}+m_{3} I_{3}
$$

with integer vector $m \in \mathbb{Z}^{3}$ satisfying $0<m_{1}<\left|m_{2}\right|<\left|m_{3}\right|$. The cases where both resonances (9) are of order 3 are called of genuine first order [39], these are the cases $1: \pm 2: \pm 3$ and $1: \pm 2: \pm 4$. Here already the cubic normal form is expected to be non-integrable, in the definite cases $1: 2: 3$ and $1: 2: 4$ this has been proven in [12].

In cases of genuine second order both resonances (9) are of order at most 4 . For $m=1: \pm 3: \pm k, k \in\{5,7,9\}$ both resonances are of the form (11) and the cubic normal form is trivial; the fourth order normal form is again expected to be non-integrable. In a 
genuine second order case where exactly one resonance is of order 3, i.e. of the form (10), the fourth order normal form is still expected to be non-integrable, but the cubic normal form $i s$ integrable and may serve as intermediate system.

If we allow for multiple eigenvalues $m_{\ell}= \pm m_{j}$, then there are additional cases $m=$ $1: \pm 1: \pm 2,1: \pm 2: \pm 2$ of genuine first order and $m=1: 1: \pm 1,1: \pm 1: \pm 3,1: \pm 3:$ \pm 3 of genuine second order [39]. In the definite case $1: 1: 2$ the cubic normal form is known to be non-integrable $[12,18]$ while for $m= \pm 1: 2: 2$ the cubic normal form may serve as intermediate system [1,25,31]; in the additional cases of genuine second order the cubic normal form is again trivial. In indefinite cases with double eigenvalues of opposite symplectic sign these may be involved in a Krein collision and leave the imaginary axis during a subordinate Hamiltonian Hopf bifurcation.

To construct generalized action angle variables for (13) we take $y=H_{m}^{2}$ itself as action, the conjugate angle $x$ then is the time along the periodic flow of the resonant oscillator. Abusing notation by also writing $y$ for the (fixed) value of (13) and dividing out the $S^{1}$ action defined by the periodic flow we obtain the reduced phase space

$$
\mathscr{P}_{y}:=\left(H_{m}^{2}\right)^{-1}(y) / S^{1}
$$

and any choice $\left(\xi_{1}, \xi_{2}, \eta_{1}, \eta_{2}\right)$ of canonical co-ordinates on $\mathscr{P}_{y}$ yields generalized action angle variables $(x, y, \xi, \eta)$. The global structure of $\mathscr{P}_{y}$ depends on $m \in \mathbb{Z}^{3}$ and can e.g. be studied using the invariants of the $S^{1}$-action $[29,39]$.

Reduction of the first non-trivial normal form leads to a two-degree-of-freedom problem on $\mathscr{P}_{y}$ and since normalization preserves symmetries a simple way to enforce integrability is to require the original Hamiltonian to admit the suitable symmetry. The resonance $m_{\ell}=m_{j}$ makes an axial $S^{1}$-symmetry an obvious candidate, but all that is needed is to prevent certain third or fourth order terms to appear in the normal form whence invariance under a discrete subgroup $\mathbb{Z}_{k}$ of $S^{1}$ suffices, with $k=2$ for third order terms, $k=3$ for fourth order terms or $k \geq 5$ just to be sure. Discrete symmetries can also provide the other cases with an integrable normal form [39], often a reversing reflection $p_{\ell} \mapsto-p_{\ell}$ is all that is needed.

\section{Higher Superintegrability}

For minimally superintegrable Hamiltonian systems, normalization of a given perturbation with respect to the unperturbed flow always removes the degeneracy-subject to a nondegeneracy condition on the intermediate system, i.e. on the perturbation. In some cases this remains true where superintegrability is not minimal, for instance in the presence of additional symmetry. However, if already the first non-trivial normal form is not integrable, then alternative ways to obtain an integrable intermediate system become important.

For a free rigid body with principal moments of inertia $I_{1}=I_{2}=I_{3}$ all motions are periodic (as every axis is a main axis of inertia); recall that we exclude the zero section $S O(3) \subseteq T^{*} S O(3)$ of equilibria. This is a maximally superintegrable system and a semiglobal chart $(x, y, \mu, \ell)$ is given by the angle $x$ measuring the rotation (= precession) about the angular momentum vector, by the length $y$ of that vector and by restricting $\mu, \ell \in \mathbb{R}^{3}$ to the symplectic fibres

$$
S_{y}^{2} \times S_{y}^{2}=\left\{(\mu, \ell) \in \mathbb{R}^{6} \mid \mu_{1}^{2}+\mu_{2}^{2}+\mu_{3}^{2}=\ell_{1}^{2}+\ell_{2}^{2}+\ell_{3}^{2}=y^{2}\right\}
$$


of the bifibration, see Fig. 1. Already quadratic force fields have averages that cannot be used as integrable intermediate system (while the linear part of a force field can not be 'seen' by a dynamically spherically symmetric top).

Coupling $m$ such tops gives rise to a superintegrable system with tori of dimension $n=m$ in $d=3 m$ degrees of freedom. Replacing some (or all) of them with Euler and Lagrange tops allows to obtain ramified $\mathbb{T}^{n}$-bundles with any $m \leq n \leq d=3 m$. Recall that imposing $d-n$ resonances on an elliptic equilibrium even allows for all $n$ between the maximal case $n=1$ and the case $n=d-1$ of minimal superintegrability.

\subsection{Perturbations That Remove the Degeneracy}

The (spatial) Kepler system is the most famous example of a maximally superintegrable system in three degrees of freedom. Some perturbed Keplerian systems like the lunar problem $[14,40]$ and the hydrogen (or Rydberg) atom in crossed fields [15, 21] display a helpful time scale phenomenon. In both cases the normal form

$$
\bar{H}=K+\varepsilon K L
$$

obtained by averaging along the Keplerian ellipses is the sum of the unperturbed $H_{0}=K$ and a scalar multiple of the product $K L$, where $L$ is the third component of the angular momentum. This special structure of $\bar{H}$ allows for a second normalization, now with respect to $L$, that results in

$$
\mathscr{H}=K+\varepsilon K L+\varepsilon^{2} H_{1}(K, L, I)
$$

with an appropriately chosen third action $I$. For the lunar problem the difference between the twice normalized $\mathscr{H}$ and the original Hamiltonian $H$ is of order $\varepsilon^{8 / 3}$ and taking $\mathscr{H}$ as intermediate system yields a Cantor family of 3-tori with three time scales (and two time scales $\varepsilon$ and $\varepsilon^{2}$ of rates of change of these freqencies) that persist into the original system [40].

Perturbations of superintegrable systems always have three time scales: the fast motion on the $n$-tori (the petals of the Fassò flowers), the slow motion on the symplectic fibres (the centres of the Fassò flowers) and the very (exponentially) slow motion of the actions (in the meadow for the Fassò flowers). Where the dynamics on the symplectic fibres of the reduced averaged system has as many time scales as degrees of freedom-slow, slower, ..., slowest (but still faster than exponentially slow) — it should be possible to construct an appropriate intermediate system [26].

\subsection{The C. Neumann Hierarchy}

The Neumann system describes a particle confined to a sphere $S^{d}$ and moving in a quadratic potential $\frac{1}{2} \sum b_{i} q_{i}^{2}$. Only the differences between the eigenvalues $b_{i}, i=0, \ldots, d$ of the linear force field have dynamical consequences and indexing equal eigenvalues $b_{\sigma}$ by $I_{\sigma}$, $\sigma=0, \ldots, \ell$ with $\left|I_{\sigma}\right|=m_{\sigma}$, the Hamiltonian is invariant under the symmetry group

$$
O\left(m_{0}\right) \times O\left(m_{1}\right) \times \cdots \times O\left(m_{\ell}\right)
$$

(in particular $d+1=m_{0}$ for $\ell=0$ where the Neumann system becomes the geodesic flow on $S^{d}$ ). Integrals of motion are the angular momenta

$$
L_{i j}=q_{i} p_{j}-q_{j} p_{i}, \quad i, j \in I_{\sigma}
$$


together with

$$
F_{\sigma}=\sum_{i \in I_{\sigma}}^{m_{\sigma}} q_{i}^{2}+\sum_{\tau \neq \sigma}^{\ell} \sum_{j \in I_{\sigma}}^{m_{\sigma}} \sum_{k \in I_{\tau}}^{m_{\tau}} \frac{L_{i j}^{2}}{b_{\sigma}-b_{\tau}}, \quad \sigma=0, \ldots, \ell .
$$

For $m_{\sigma}=1$ the latter turn into the Uhlenbeck integrals; the Neumann system is integrable even if all eigenvalues are different from each other. From now on we assume $m_{\sigma} \geq 2$ for all $\sigma=0, \ldots, \ell$ (see [19] for the general case). Note that the symmetry group (14) is commutative if $m_{\sigma}=2$ for all $\sigma$ and almost all quasi-periodic motions have $d$ frequencies.

For $m_{\sigma} \geq 3$ the factor $O\left(m_{\sigma}\right)$ is non-commutative and enforces superintegrability. Reducing (14) leads to the Rosochatius system on $S^{\ell}$ and action angle variables $(u, v) \in$ $\mathbb{T}^{\ell} \times \mathbb{R}^{\ell}$ of this integrable system serve as starting point for the construction of generalized action angle variables. The additional actions are the total angular momenta

$$
y_{\sigma}=\left(\sum_{i, j \in I_{\sigma}}^{m_{\sigma}} L_{i j}^{2}\right)^{\frac{1}{2}}, \quad \sigma=0, \ldots, \ell
$$

with conjugate angles $x_{\sigma}$ measuring the rotation in the plane singled out by $L_{i j}, i, j \in I_{\sigma}$ (for $m_{\sigma}=3$ this is the usual plane perpendicular to the angular momentum vector). The symplectic leaves of the bifibration are the co-adjoint orbits

$$
G_{m_{0} 2} \times G_{m_{1} 2} \times \cdots \times G_{m_{\ell} 2}
$$

of products of Graßmannians

$$
G_{m_{\sigma} 2}=O\left(m_{\sigma}\right) / S O(2) \times O\left(m_{\sigma}-2\right)
$$

parametrising the oriented planes in $\mathbb{R}^{m_{\sigma}}$. Any choice of canonical co-ordinates $\left(w_{k}, z_{k}\right)$, $k=1, \ldots, \sum\left(m_{\sigma}-2\right)$ defines generalized action angle variables $(u, x, v, y, w, z)$ for the Neumann system. Note that the torus dimension is $n=2 \ell+1$-even dimensions $n$ can be achieved if also allowing for $m_{\sigma}=1$, i.e. eigenvalues that are distinct from all other eigenvalues [19].

Averaging a perturbation of the Neumann system along the quasi-periodic $n$-tori introduces a $\mathbb{T}^{n}$-symmetry, the reduction of which leads to a Hamiltonian system on (15), in $d-n=\sum\left(m_{\sigma}-2\right)$ degrees of freedom. In the minimal case $m_{\sigma}=3$ (all other $m_{\tau}=2$ ) the product (15) has a single non-trivial factor $G_{m_{\sigma} 2} \cong S_{y_{\sigma}}^{2}$, the sphere of possible directions of the angular momentum vector. In the maximal case $\ell=0, m_{0}=d+1$ we have a single Graßmannian as well.

\section{Conclusions}

KAM theory is about integrable systems - under strong non-resonance conditions their quasi-periodic solutions persist under sufficiently small perturbations. This does not directly apply to superintegrable Hamiltonian systems. Here it is the ramified $d$-torus bundle of an integrable intermediate system that is Cantorised by the perturbation, with information not only from the unperturbed system but also from the perturbation itself.

In the minimally superintegrable case of $d+1$ conserved quantities the perturbation analysis consists of three steps: compute a normal form that serves as intermediate system, 
study how the dynamics of the intermediate system structures the phase space (organized by the fast $(d-1)$-tori, the relative equilibria) and check the necessary non-degeneracy conditions concerning both the superintegrable system and the reduced intermediate system.

Where an integrable intermediate system can be found in cases with $n \leq d-2$ this threestep-programme still applies. However, while this is automatic in the case $n=d-1$ of minimal superintegrability, additional properties are needed when $n \leq d-2$-for instance symmetries of the (perturbed) system or $d-n$ time scales within the perturbed dynamics (apart from the multi-periodic fast angles and the exponentially slow variation of the actions). In principle, the perturbation problem may lead to any Hamiltonian system in $d-n$ degrees of freedom.

The resonant lattices studied in [42] are maximally superintegrable; reducing the normal form with respect to the periodic flow leads to a quotient $S^{2 d-1} / S^{1}$ where the $S^{1}$-action is free only for the case $1: 1: 1: \ldots: 1$. In the other cases the $S^{1}$-action is locally free and the (discrete) isotropy groups lead to singularities of the reduced phase space: the Hamiltonian system in $d-1$ degrees of freedom is not defined on a symplectic manifold but on a Poisson space. For indefinite lattices the reduced phase space is furthermore not compact.

Every resonance (9) of an elliptic equilibrium increases the co-dimension of that Hamiltonian system within the universe of all Hamiltonian systems. For instance, two parameters are needed to generically encounter an equilibrium with quadratic part (13) of the Hamiltonian; both parameters detune the frequency ratio. However, if these are normal resonances of invariant tori, then the complete unfolding may be encountered within a single (generic) Hamiltonian system.

In this way the 3-parameter families of invariant 3-tori in six degrees of freedom can have 1-parameter subfamilies with normal behaviour (13). A successful application of KAM theory would eventually lead to 1-parameter Cantor families of such 3-tori, organizing the Cantorised ramified torus bundle of the unfolding [29]. Clearly, the development of such a theory has to start with elliptic equilibria of Hamiltonian systems depending on external parameters.

Perturbing resonant $d$-tori of a (non-degenerate) integrable system resembles perturbing a superintegrable system. Letting $d-n$ denote the number of independent (internal) resonances, normalizing with respect to the quasi-periodic flow with $n$ frequencies yields a Hamiltonian that admits a local $\mathbb{T}^{n}$-action and can be reduced to $d-n$ degrees of freedom with a cylinder $\mathbb{T}^{d-n} \times \mathbb{R}^{d-n}$ as phase space. For $n=d-1$ the resulting family of integrable intermediate systems can in particular be used to identify those $(d-1)$-tori foliating the resonant $d$-tori that persist the perturbation [9].

The Cantor families of such $(d-1)$-tori organize the phase space and persistently undergo bifurcations of co-dimension up to $d-2$. Similar approaches $[11,13]$ with $n \leq d-2$ restrict to elliptic and hyperbolic tori, the minimal number of which is expected to coincide with the minimal number $n+1$ of critical points of a smooth function on $\mathbb{T}^{n}$. A better understanding of perturbed superintegrable systems would entail a better understanding of the perturbed dynamics near resonant tori.

Acknowledgements I thank Henk Broer, Antonella Marchesiello, Giuseppe Pucacco and Ferdinand Verhulst for helpful discussions and the referees for their guiding remarks.

Open Access This article is distributed under the terms of the Creative Commons Attribution License which permits any use, distribution, and reproduction in any medium, provided the original author(s) and the source are credited. 


\section{References}

1. van der Aa, E., Verhulst, F.: Asymptotic integrability and periodic solutions of a Hamiltonian system in $1: 2: 2$-resonance. SIAM J. Math. Anal. 15(5), 890-911 (1984)

2. Arnol'd, V.I.: Proof of a theorem of A.N. Kolmogorov on the invariance of quasi-periodic motions under small perturbations of the Hamiltonian. Russ. Math. Surv. 18(5), 9-36 (1963)

3. Arnol'd, V.I.: Small denominators and problems of stability of motion in classical and celestial mechanics. Russ. Math. Surv. 18(6), 85-191 (1963)

4. Arnol'd, V.I., Kozlov, V.V., Neishtadt, A.I.: Mathematical aspects of classical and celestial mechanics. In: Arnol'd, V.I. (ed.) Dynamical Systems III. Springer, Berlin (1988)

5. Binney, J.: Resonant excitation of motion perpendicular to galactic planes. Mon. Not. R. Astron. Soc. 196, 455-467 (1981)

6. Binney, J., Tremaine, S.: Galactic Dynamics. Princeton University Press, Princeton (2008)

7. Broer, H.W., Huitema, G.B., Takens, F.: Unfoldings of quasi-periodic tori. Mem. Am. Math. Soc. 83 \#421, 1-82 (1990)

8. Broer, H.W., Huitema, G.B., Sevryuk, M.B.: Quasi-Periodic Motions in Families of Dynamical Systems: Order Amidst Chaos. LNM, vol. 1645. Springer, Berlin (1996)

9. Broer, H.W., Hanßmann, H., You, J.: On the destruction of resonant Lagrangean tori in Hamiltonian systems. In: Johann, A., et al. (eds.) Recent Trends in Dynamical Systems, München 2012. Proceedings in Mathematics \& Statistics, vol. 35, pp. 317-333. Springer, Berlin (2013)

10. Broer, H.W., Hanßmann, H., Wagener, F.O.O.: Quasi-periodic bifurcation theory: the geometry of KAM. (in preparation)

11. Cheng, C.-Q., Wang, S.: The surviving of lower dimensional tori from a resonant torus of Hamiltonian systems. J. Differ. Equ. 155(2), 311-326 (1999)

12. Christov, O.: Non-integrability of first order resonances of Hamiltonian systems in three degrees of freedom. Celest. Mech. Dyn. Astron. 112, 147-167 (2012)

13. Cong, F., Küpper, T., Li, Y., You, J.: KAM-type theorem on resonant surfaces for nearly integrable Hamiltonian systems. J. Nonlinear Sci. 10, 49-68 (2000)

14. Cushman, R.: A survey of normalization techniques applied to perturbed Keplerian systems. Dyn. Rep. New Ser. 1, 54-112 (1992)

15. Cushman, R., Sadovskií, D.A.: Monodromy in the hydrogen atom in crossed fields. Physica D 142, 166-196 (1998)

16. Dazord, P., Delzant, T.: Le problème général des variables actions-angles. J. Differ. Geom. 26, 223-251 (1987)

17. Duistermaat, J.J.: Bifurcations of periodic solutions near equilibrium points of Hamiltonian systems. In: Salvadori, L. (ed.) Bifurcation Theory and Applications, Montecatini 1983. LNM, vol. 1057, pp. 57-105. Springer, Berlin (1984)

18. Duistermaat, J.J.: Non-integrability of the $1: 1:$ 2-resonance. Ergod. Theory Dyn. Syst. 4, 553-568 (1984)

19. Dullin, H.A., Hanßmann, H.: The degenerate C. Neumann system I: symmetry reduction and separation. In: Čap, A., et al. (eds.) Finite Dimensional Integrable Systems, Dynamics, and Lie Theoretic Methods in Geometry and Mathematical Physics, Jena 2011. Cent. Eur. J. Math. 10, pp. 1627-1654 (2012)

20. Dumas, H.S.: The KAM Story: a Friendly Introduction to the Content, History, and Significance of Classical Kolmogorov-Arnold-Moser Theory. World Scientific, Singapore (2014)

21. Efstathiou, K.: Metamorphoses of Hamiltonian Systems with Symmetries. LNM, vol. 1864. Springer, Berlin (2005)

22. Fassò, F.: The Euler-Poinsot top: a non-commutatively integrable system without global action-angle coordinates. Z. Angew. Math. Phys. 47, 953-976 (1996)

23. Fassò, F.: Superintegrable Hamiltonian systems: geometry and perturbation. In: Gaeta, G. (ed.) Symmetry and Perturbation Theory, Cala Gonone 2004. Acta Appl. Math. 87, pp. 93-121 (2005)

24. Gerhard, O., Binney, J.: Triaxial galaxies containing massive black holes or central density cusps. Mon. Not. R. Astron. Soc. 216, 467-502 (1985)

25. Haller, G.: Chaos Near Resonance. AMS, vol. 138. Springer, Berlin (1999)

26. Han, Y., Li, Y., Yi, Y.: Invariant tori in Hamiltonian systems with high order proper degeneracy. Ann. Inst. Henri Poincaré 10, 1419-1436 (2010)

27. Hanßmann, H.: Quasi-periodic motions of a rigid body I-quadratic Hamiltonians on the sphere with a distinguished parameter. Regul. Chaotic Dyn. 2(2), 41-57 (1997)

28. Hanßmann, H.: The quasi-periodic centre-saddle bifurcation. J. Differ. Equ. 142(2), 305-370 (1998)

29. Hanßmann, H.: Local and Semi-Local Bifurcations in Hamiltonian Dynamical Systems-Results and Examples. LNM, vol. 1893. Springer, Berlin (2007)

30. Hanßmann, H.: Non-degeneracy conditions in KAM theory. Indag. Math. 22, 241-256 (2011) 
31. Kummer, M.: On resonant Hamiltonian systems with finitely many degrees of freedom. In: Sáenz, A.V., Zachary, W.W., Cawley, R. (eds.) Local and Global Methods of Nonlinear Dynamics, Silver Spring 1984. Lect. Notes Phys., vol. 252, pp. 19-31. Springer, Berlin (1986)

32. Markus, L., Meyer, K.R.: Generic Hamiltonian dynamical systems are neither integrable nor ergodic. Mem. Am. Math. Soc. \#144, 1-52 (1974)

33. Mazzocco, M.: KAM theorem for generic analytic perturbations of the Euler system. Z. Angew. Math. Phys. 48, 193-219 (1997)

34. Mishchenko, A.S., Fomenko, A.T.: Generalized Liouville method of integration of Hamiltonian systems. Funct. Anal. Appl. 12(2), 113-121 (1978)

35. Moser, J.: Convergent series expansion for quasi-periodic motions. Math. Ann. 169(1), 136-176 (1967)

36. Nekhoroshev, N.N.: Action-angle variables and their generalizations. Trans. Mosc. Math. Soc. 26, 180198 (1972)

37. Papaphilippou, Y., Laskar, J.: Global dynamics of triaxial galactic models through frequency map analysis. Astron. Astrophys. 329, 451-481 (1998)

38. Persic, M., Salucci, P., Stel, F.: The universal rotation curve of spiral galaxies: I. The dark matter connection. Mon. Not. R. Astron. Soc. 281, 27-47 (1996)

39. Sanders, J.A., Verhulst, F., Murdock, J.: Averaging Methods in Nonlinear Dynamical Systems, 2nd edn. AMS, vol. 59. Springer, Berlin (2007)

40. Sommer, B.S.: A KAM Theorem for the Spatial Lunar Problem. Ph.D. thesis, RWTH Aachen (2003)

41. Touma, J., Tremaine, S.: A map for eccentric orbits in non-axisymmetric potentials. Mon. Not. R. Astron. Soc. 292, 905-919 (1997)

42. Verhulst, F.: Integrability and mode-interaction for Hamiltonian normal forms. Acta Appl. Math. (2015). doi:10.1007/s10440-014-9998-5

43. de Zeeuw, T.: Dynamical models for axisymmetric and triaxial galaxies. In: de Zeeuw, T. (ed.) Structure and Dynamics of Elliptical Galaxies. IAU Symp., vol. 127, pp. 271-290. Reidel, Dordrecht (1987) 